\title{
Human Face Identification based on Optimal Sparse Features
}

\author{
M. Risheek Sharma, K. Akhil Vardhan, K. Sravan Kumar, B. Koteswarrao, Shijin Kumar P. S.
}

\begin{abstract}
Security of human being is an important aspect in the context of data communication. To maintain security, technology is being developed from alpha-numeric passwords to biometric scanners. Recent advancement in security is the user authentication using face recognition. But the flaws in existing face recognition systems are yet to be addressed. This paper discusses solutions to the issues encountered by face recognition systems. Sparsity based classification is performed in this work. This method can handle errors occurs due to compress in and occlusion in a robust manner. We suggest a comprehensive classification algorithm characterized by sparse representation and 11 -minimization. In this method, the feature points and selection of features are not critical. The effect of change in occlusion can be easily addressed by using this optimal sparse representation based classification (OSRC) algorithm.
\end{abstract}

Keywords: Face Detection, Sparsity, Optimal Sparse Representation based Classification, $l_{1}$-minimization.

\section{INTRODUCTION}

Face recognition can be considered as a difficult and hot study subjects in last few years. We can discover numerous methods to identify and verify persons like passwords, pin number, fingerprint, scanners etc. Face recognition provides many benefits, since it is a passive scheme for verification [1]. Most of the existing face identification methods are not precise and their efficiency can be affected by several variables. Consider the image of a person taken under different conditions. The image of same person will vary from time to time. The efficacy of existing algorithms is adversely affected from obstructions; improper lighting [3],

Revised Manuscript Received on December 30, 2019.

* Correspondence Author

M. Risheek Sharma, Pursuing Bachelor of Technology, Department of Electronics and Communication Engineering, Marri Laxman Reddy Institute of Technology and Management, Dundigal, Hyderabad (Telangana) India. E-mail: risheek1512@gmail.com

K. Akhil Vardhan, Pursuing Bachelor of Technology, Department of Electronics and Communication Engineering, Marri Laxman Reddy Institute of Technology and Management, Dundigal, Hyderabad (Telangana) India. E-mail: akhilvardhan.kondeti@gmail.com.

K. Sravan Kumar, Pursuing Bachelor of Technology, Department of Electronics and Communication Engineering, Marri Laxman Reddy Institute of Technology and Management, Dundigal, Hyderabad (Telangana) India. E-mail: sravankarrena444@gmail.com

B. Koteswarrao, Pursuing PhD Degree, Department of Electronics and Communication Engineering, Marri Laxman Reddy Institute of Technology and Management, Dundigal, Hyderabad (Telangana) India. E-mail: ksrao123@mlritm.acin

Shijin Kumar P. S.*, Associate Professor, Department of Electronics and Communication Engineering, Marri Laxman Reddy Institute of Technology and Management, Dundigal, Hyderabad (Telangana) India. E-mail: shijinkumarps@yahoo.com

(C) The Authors. Published by Blue Eyes Intelligence Engineering and Sciences Publication (BEIESP). This is an open access article under the CC BY-NC-ND license (http://creativecommons.org/licenses/by-nc-nd/4.0/) expression change and varying pose [4]. Traditional face recognition algorithms adhere to geometry [2] based approach or piecewise [6] appearance oriented approach.

For face recognition, these techniques employ raw facial images. In this paper we introduce optimal sparse technique for recognizing faces. It is a fresh approach of acquiring sparse signals and representing them. Chu et al [11]

developed a geometry based feature extraction technique for the recognition of facial images in severe conditions. This method provided a recognition rate of $96.4 \%$. The computational time is high and it is sensitive to illumination and poses variation. Wright et al [12] proposed a simple face recognition system based on sparse representation. This method is highly robust against corruption due to occlusion. The recognition rate obtained was $98.3 \%$. Anand et al [13] used SVM for the recognition of faces using SURF features. The database used was Yale faces and the recognition rate was 97.8\%. Riddhi et al [4] compared PCA and LDA techniques for the recognition of faces. They used Yale Faces dataset and the accuracy obtained was $98.18 \%$.

We propose a comprehensive classification algorithm characterized by sparse representation and $\mathrm{l}_{1}$-minimization. In this method, the feature points and selection of features are not critical. The effect of change in occlusion can be easily addressed by using this optimal sparse representation based classification (OSRC) algorithm. Remainder of this paper is arranged in three sections. Section II displays the proposed face recognition method based on sparse features. Section III displays the experimental results obtained using the proposed methodology. Finally, section IV concludes the paper.

\section{METHODOLOGY}

The block diagram of proposed face recognition system is based on optimal sparse features is illustrated in Fig.1. This method is efficient and fast. 


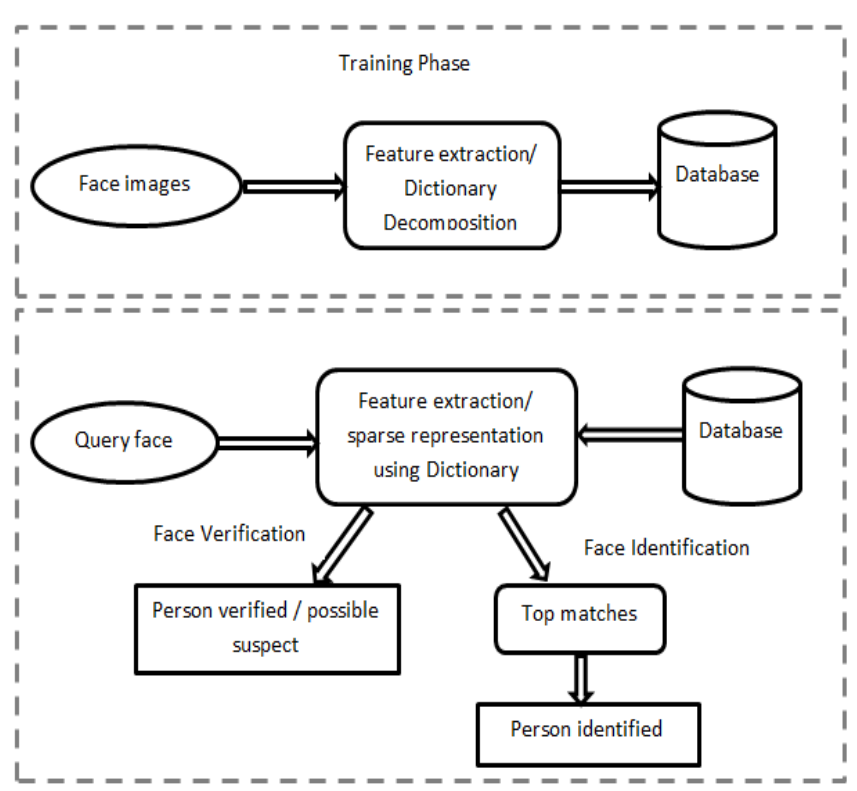

Fig.1. Block Diagram of Proposed Method.

This work's initial objective is to show the pixels at reduced sample rates than that of Shannon-Nyquist link [7]. Fundamental concept of this structure is to see a fresh test picture using linear sparse mixture of train images. This technique looks for the closest linear picture depiction from all training samples. We consider overall information from both test samples and train samples. If sufficient samples are present from individual classes, the image of test sample will then be shown as a linear consolidation of same class samples and test sample image for a limited number of classes. The geometry of sparse representation using $l_{1}$-minimization is given in Fig.2.

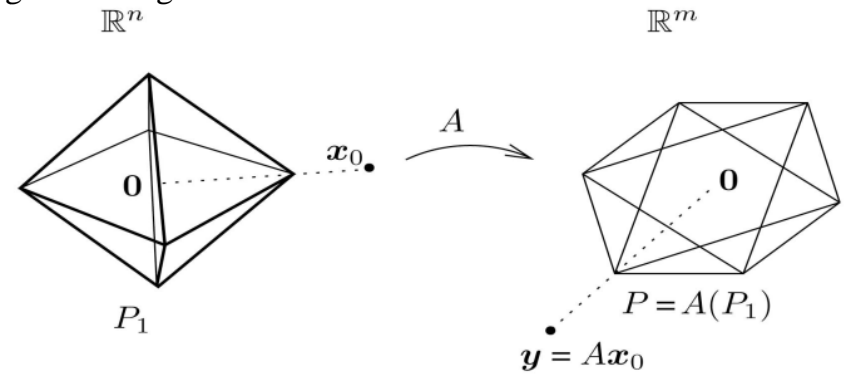

Fig. 2 Geometry of sparse represesntation using $l_{1}$-minimization

By applying sparse and $l_{1}$-minimization, stored face images are retrieved properly. The equations to form a sparse vector are acquired through $l_{1}$ normalization method. Classification [8] is performed using sparse vectors and during recovery we get a face images. Facial identification problem is determined in order to select the class of given test sample. A picture matrix is recognized for grey scale image of size $i \times j$. Transformed vector is obtained by concatenating its columns into a vector and subsequently a vector of image $v$ is generated. Each $m^{\text {th }}$ class vector of image is arranged as a column of matrix. The issues in this representation are resolved through by linear programming techniques in polynomial times. If the solution is known to be quite small, even more effective techniques are accessible.

\section{EXPERIMENTAL RESULTS}

In this section, the recognition rate of the proposed face identification system is calculated and the results are analyzed. The dataset used for this experiment consist of 400 different facial images. Sample images from the dataset are shown in Fig.3 and Fig.4. As shown in the figures, images are having different pose, illumination and expressions.

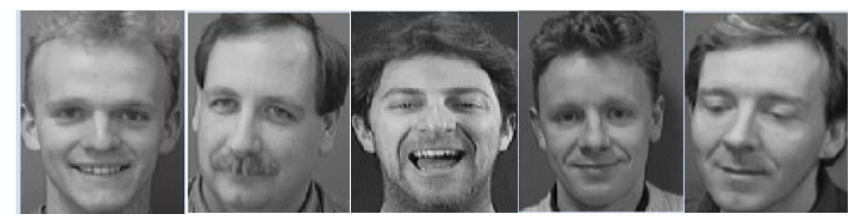

Fig. 3 Sample train images

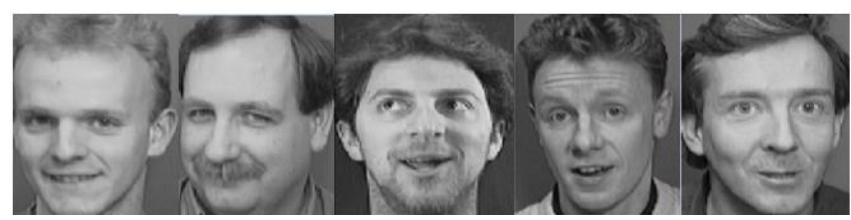

Fig.4 Sample test images

Recognition rate is calculated using the ratio of correctly classified images to the total number of images used for testing. Images in the dataset are divided into two categories. Train category consists of 200 images and test category consists of 200 images. 5 image samples of an individual are present in each category. Initially, training is performed using all 200 images in the train category. Testing is was performed using individual images from the test category [9]. The image format used in this experiment is "portable gray map" (PGM) and the size of the images is $92 \times 112$. Each image file requires $10 \mathrm{~Kb}$ of storage space and the resolution is $72 \mathrm{dpi}$. Face detection output is illustrated in Fig.5.

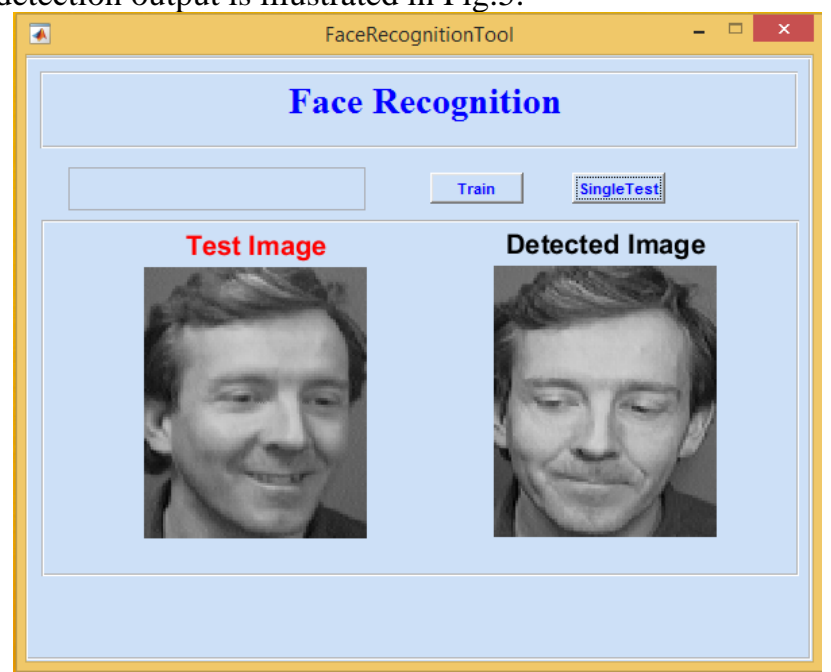

Fig.5. Face Detection Result

Existing Sparse Representation based Classification (SRC) provides a recognition rate of $94.7 \%$. Optimal sparse representation based classification (OSRC) algorithm provides a recognition rate [10] of $99.17 \%$. Not only in recognition rate, but also there is some improvement in computational time.

Table 1 Comparison between SRC and OSRC

\begin{tabular}{ccccc}
\hline Image Set & \multicolumn{2}{c}{ Recognition Rate (\%) } & \multicolumn{2}{c}{ Time (s) } \\
\hline & SRC & OSRC & SRC & OSRC \\
\hline 1 & 89.16 & 98.15 & 1.49 & 0.48 \\
2 & 94.11 & 98.47 & 3.17 & 0.52
\end{tabular}




\begin{tabular}{ccccc}
3 & 94.06 & 99.27 & 4.33 & 0.68 \\
4 & 98.13 & 100 & 5.13 & 0.71 \\
5 & 98.04 & 100 & 5.66 & 0.78 \\
\hline
\end{tabular}

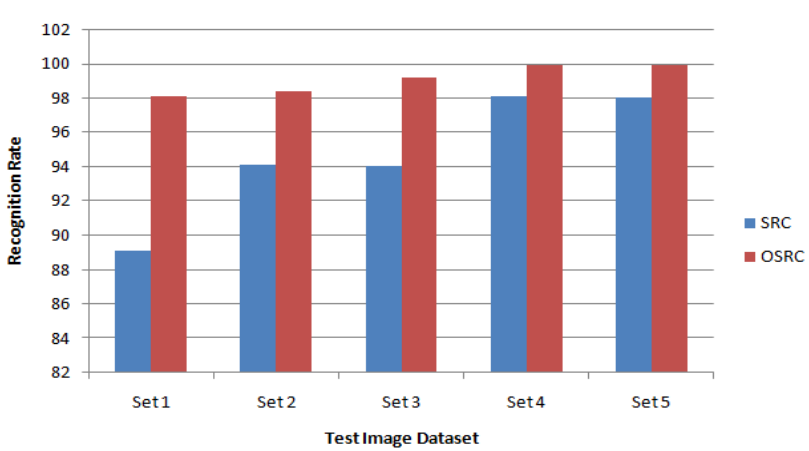

Fig.6. Comparison of Recognition Rate (SRC Vs OSRC)

Though the recognition rate is almost equal for both algorithms, there is a noticeable reduction in computational time. OSRC is faster than normal SRC. Table 1 explains the variation in recognition rate and computational time for various test image sets. Fig. 6 displays a comparison between the recognition rate of SRC and OSRC. Fig. 7 displays a comparison between the computational time of SRC and OSRC.

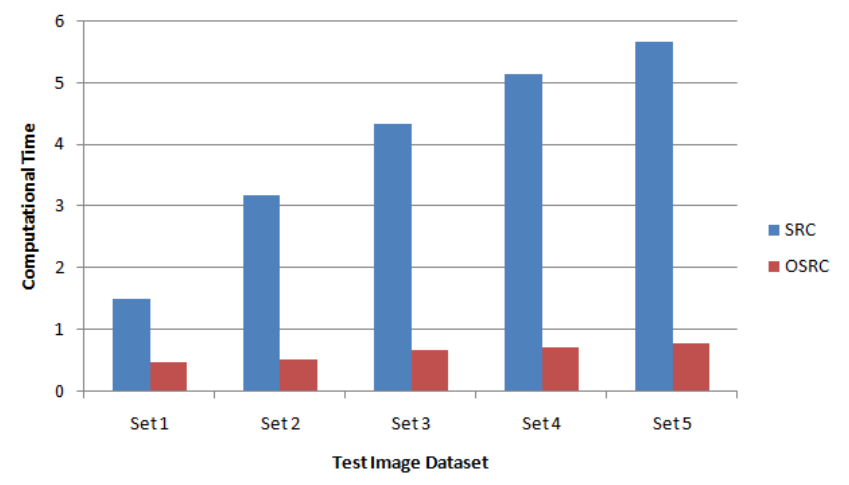

Fig.7. Comparison of Computational Time (SRC Vs OSRC)

The recognition rate of proposed system is compared with existing methodologies and it has been found that the proposed system outperforms all existing methods. The comparison results are given in Table 2 and Fig.8.

Table 2 Comparison of Recognition Rate with Existing Methods

\begin{tabular}{cc}
\hline Methodology & Recognition Rate (\%) \\
\hline Geometry Based [11] & 96.14 \\
Sparse Features [12] & 98.30 \\
SURF+SVM [13] & 97.80 \\
PCA+LDA [14] & 98.18 \\
OSRC (Proposed) & 99.17 \\
\hline
\end{tabular}

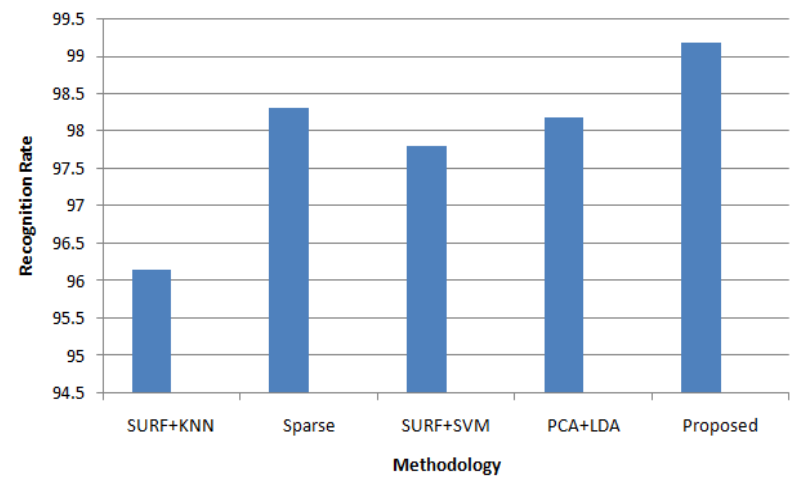

Fig.8. Comparison of Recognition Rate

\section{CONCLUSION}

We have shown in this paper about the importance and growth of facial recognition system. Although, this is an important step forward, much more needs to be achieved in order to improve the computational time. Though the rate of acceptance of both SRC and OSRC are similar, but there is a considerable reduction in computational time. This paper screened a single sample per topic problem and the findings with recognition rate are satisfactory. OSRC algorithm can also be developed for small samples, higher-dimensional images and larger numbers of classes. In this work, we tested the proposed algorithm with gray scale '.pgm' image database of different person in various occlusion and pose. Major peculiarity of this algorithm is the ability to adapt for non linear distribution of train images. The algorithm has to be operated in less constrained condition to obtain optimal results. In future, we are planning to conduct experiment on other databases so as to verify whether the implementation of this face recognition system work on real time applications. Further it is possible to combine sparse representation system with other face recognition algorithms. Including feature matching techniques along with OSRC algorithm can yield better recognition rate and it is our prime focus in the future.

\section{REFERENCES}

1. Samal, A., \& Iyengar, P. A. Automatic recognition and analysis of human faces and facial expressions: A survey. Pattern recognition, 25(1), (1992), 65-77.

2. Kotsia, I., \& Pitas, I. Facial expression recognition in image sequences using geometric deformation features and support vector machines. IEEE transactions on image processing, 16(1), (2006), 172-187.

3. Sinha, P., Balas, B., Ostrovsky, Y., \& Russell, R. Face recognition by humans: Nineteen results all computer vision researchers should know about. Proceedings of the IEEE, 94(11), (2006). 1948-1962.

4. Zhao, W., \& Chellappa, R. Image-based face recognition: Issues and methods. Optical engineering-New York-marcel dekker incorporated, 78, (2002). 375-402.

5. Samaria, F. S. (1994). Face recognition using hidden Markov models (Doctoral dissertation, University of Cambridge).

6. Lu, X. (2003). Image analysis for face recognition. Personal notes, 36.

7. Bachu, S., \& Teja, N. R. Fuzzy Holoentropy-Based Adaptive Inter-Prediction Mode Selection for H. 264 Video Coding. International Journal of Mobile Computing and Multimedia Communications (IJMCMC), 10(2), (2019), 42-60.

8. Shijin Kumar P.S and Sudhan M.B. "A Hybrid Framework for Brain Tumor Detection and Classification using Neural Network" ARPN Journal of Engineering and Applied Sciences 13(24) (2018): pp.9631-9636. 
9. Bachu, S., \& Achari, K. M. Adaptive order search and tangent-weighted trade-off for motion estimation in H. 264. Journal of King Saud University-Computer and Information Sciences, 30(2), (2018), 249-258.

10. Shijin Kumar P.S and V. S. Dharun. "Combination of Fuzzy C-means Clustering and Texture Pattern Matrix for Brain MRI Segmentation." Biomedical Research 28(5) (2017): pp.2046-2049.

11. Chu, D., \& Thye, G. S. (2010). A new and fast implementation for null space based linear discriminant analysis. Pattern Recognition, 43(4), 1373-1379.

12. Wright, A. Yang, A. Ganesh, S. Sastry and Y. Ma, Robust face recognition via sparse representation, IEEE Trans. Pattern Anal. Mach. Intell. 31(2) (2009) 210-227.

13. Anand, B., \& Shah, M. P. K. (2016). Face recognition using SURF features and SVM classifier. International Journal of Electronics Engineering Research, 8(1), 1-8.

14. Vyas, R. A., \& Shah, S. M. (2017). Comparision of PCA and LDA techniques for face recognition feature based extraction with accuracy enhancement. International Research Journal of Engineering and Technology (IRJET), 4(6), 3332-3336.

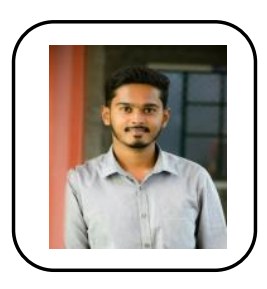

\section{AUTHORS PROFILE}

M. Risheek Sharma is pursuing Bachelor of Technology in Electronics and Communication Engineering from Marri Laxman Reddy Institute of Technology and Management., Hyderabad. He published research papers in international journals and conferences. His areas of interests are image processing, computer networking and security.

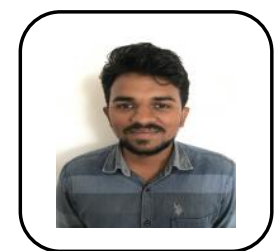

K. Akhil Vardhan is pursuing Bachelor of Technology in Electronics and Communication Engineering from Marri Laxman Reddy Institute of Technology and Management., Hyderabad. He published research papers in international journals and conferences. His areas of interests are image processing, computer networking and VLSI.

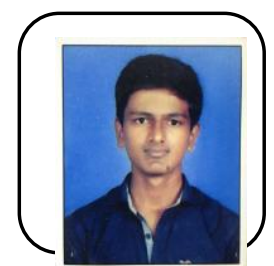

K. Sravan Kumar is pursuing Bachelor of Technology in Electronics and Communication Engineering from Marri Laxman Reddy Institute of Technology and Management., Hyderabad. $\mathrm{He}$ published research papers in international journals and conferences. His areas of interests are image processing, computer networking and VLSI.

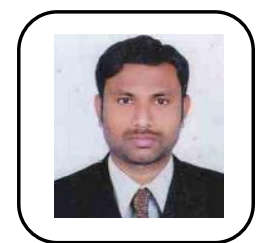

B. Koteswarrao is pursuing his $\mathrm{PhD}$ Degree in Electronics and Communication Engineering from JNTUH. He obtained his B.Tech degree in Electronics and Communication Engineering from JNTUH in the year 2006. Currently he is working as Associate Professor in the Department of Electronics and Communication Engineering at Marri Laxman Reddy Institute of Technology and Management, Hyderabad. He has more than 10 years of teaching experience. His areas of interest include Image Processing, VLSI and Machine Learning. He has more than 5 publications in international journals. He is a life member of ISTE.

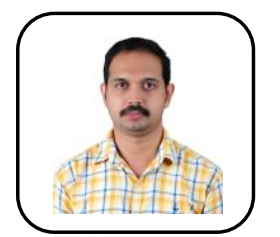

Dr. Shijin Kumar P. S. obtained his PhD Degree in Electronics and Communication Engineering from Noorul Islam University, in 2018 and M.E. in Communication Systems from Anna University in 2009. He obtained his B.Tech degree in Electronics and Communication Engineering from University of Kerala in the year 2006. Currently he is working as Associate Professor in the Department of Electronics and Communication Engineering at Marri Laxman Reddy Institute of Technology and Management, Hyderabad. He has more than 10 years of teaching experience and 1 year Industrial Experience. His area of interest includes Image Processing, Embedded Systems and Machine Learning. He has more than 10 publications in international journals and conference proceedings. He is a life member of ISTE. 